\title{
Mutual Coupling Between Two Identical Planar Inverted-F Antennas
}

Thaysen, Jesper

\section{Published in:}

Proceedings of IEEE Antennas and Propagation Society International Symposium

Link to article, DOI:

10.1109/APS.2002.1017031

Publication date:

2002

Document Version

Publisher's PDF, also known as Version of record

Link back to DTU Orbit

Citation (APA):

Thaysen, J. (2002). Mutual Coupling Between Two Identical Planar Inverted-F Antennas. In Proceedings of IEEE Antennas and Propagation Society International Symposium (Vol. 4, pp. 504-507). IEEE. https://doi.org/10.1109/APS.2002.1017031

\section{General rights}

Copyright and moral rights for the publications made accessible in the public portal are retained by the authors and/or other copyright owners and it is a condition of accessing publications that users recognise and abide by the legal requirements associated with these rights.

- Users may download and print one copy of any publication from the public portal for the purpose of private study or research.

- You may not further distribute the material or use it for any profit-making activity or commercial gain

- You may freely distribute the URL identifying the publication in the public portal

If you believe that this document breaches copyright please contact us providing details, and we will remove access to the work immediately and investigate your claim 


\title{
Mutual Coupling Between Two Identical Planar Inverted-F Antennas
}

\author{
J. Thaysen \\ Technical University of Denmark / Nokia Mobile Phones \\ jth@oersted.dtu.dk
}

I. INTRODUCTION

The Planar Inverted-F Antenna (PIFA) is widely used for cellular phones due to the compactness and size [1]. The demand for communication devices for personal communication systems has led to a constant search for ways to reduce the antenna dimensions. Applications such as triple band antennas, Bluetooth antennas, and antennas for Global Position System are emerging and thus the complexity and the requirements to the antenna system continue to increase. It is expected that high isolation between two or more frequency bands is essential in many future applications. The task is complicated by the fact that the overall size of the mobile phone and the frequency separation between the different bands continue to decrease. In order to meet these demands, physically small antenna elements with low coupling are required.

The objective of this paper is to present the results of numerical investigations of the coupling between two equal PIFA antennas. To analyse the antenna, the method of moment computer program, IE3D, was used to predict the performance of the antennas in terms of the scattering parameters. The simulated results for the PIFA located above a finite ground plane are compared to the corresponding experimental results to show the validity of this approach.

The main objective of this research is to explain the coupling between two equal PIFA antennas. Symmetrical as well as unsymmetrical coupling scenarios using two identical PIFAs located close to each other is investigated in order to determine the mutual coupling versus distance for fixed orientations, and mutual coupling versus rotation of the antennas for fixed distance. The results illustrate that the orientation and the location could be optimised to obtain minimum coupling.

\section{ANTENNA CONFIGURATION}

The PIFA investigated consists of a $40 \times 1 \mathrm{~mm}^{2}$ patch located $15 \mathrm{~mm}$ above an infinite perfect conducting ground plane. The feed line is located $13 \mathrm{~mm}$ from the edge where a $90^{\circ}$ bend forms the short circuit to the ground plane.

In the first two setups the PIFAs are vertically separated by $10 \mathrm{~mm}$, with a horizontal distance, $d$, that is defined so that $d$ is positive in the case illustrated in the vignette in Figure 2 (a). First, both antennas are fed in the right ends, hereafter called $(0 ; 0)$, and second they are fed in the opposite ends $(0 ; 180)$, as illustrated in the vignette in Figure 2 (a) and 3 (a) respectively. The third setup consists of two PIFAs located in line with a distance of $200 \mathrm{~mm}$ between the geometrical centres of the antennas. The geometrical centre is used since it is a simple measure. Another measure could be the phase centre of the antenna; this point is however not easy to identify for a PIFA [2]. In order to determine the coupling in different setups the two antennas are rotated counter clockwise around the geometrical centre in eight equally steps, each $45^{\circ}$. This gives 64 different setups, which reduces to 26 due to symmetry.

\section{VERIFICATION OF THE SIMULATED RESULTS}

In order to verify the validity of the presented results. that are obtained using IE3D, simulations are made to check the convergence. In all the cases transverse edge cells are used to make the simulations more accurate [3]. Converged results are obtained with 20 cells per wavelength. Subsequently 20 cells and transverse edge cells are used.

In Figure 1, the simulated reflection coefficient is compared to the corresponding measurements made on a PIFA located on a $40 \times 100 \mathrm{~mm}^{2}$ ground plane. The comparison yields very good agreements. At a resonant frequency of $1.89 \mathrm{GHz}$ the simulated bandwidth $\left(\left|\mathrm{S}_{11}\right|<-6 \mathrm{~dB}\right)$ is $12 \%$, which is slightly lower than for the measured result ( $14 \%$ ). The observed deviation is mainly due to the cable used in the measurements and the simulated ideal assumption, i.e., lossless and in free space, as compared to the ROHACELL material used for the prototype.

0-7803-7330-8/02\$17.00@2002 IEEE 


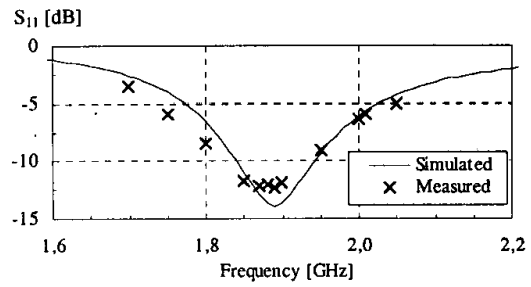

Figure 1. Measured and simulated reflection coefficient for the pifa above a $40 \times 100 \mathrm{~mm}^{2}$ ground plane.

IV. NUMERICAL RESULTS

Simulations on two equal PIFAs located on an infinite ground plane are carried out in order to determine the mutual coupling.

A. Mutual Coupling versus Distance for Fixed Parallel Orientations: Figure 2 (a) shows the simulated S-parameters as a function of the horizontal distance, $d$. The highest coupling, $\mathrm{S}_{21}=-4.8 \mathrm{~dB}$ is obtained for $d=0$. A symmetrical rolloff is observed when the distance is increased; this seems reasonably due to the symmetry. The impedance match for the two antennas shown in Figure 2 (a) also indicates symmetry, at $d= \pm 13 \mathrm{~mm}$ where the shorting point is above the feed line the impedance match is worst.

The resonant frequency, shown in Figure $2(\mathrm{~b})$, is only altered slightly as a function of distance. The largest deviation is obtained at zero horizontal distance between the open end of one of the antennas and the feed line of the other. Here the resonant frequency is shifted 30 $\mathrm{MHz}$ compared to the result that is obtained for a single antenna. At $d=0 \mathrm{~mm}$ the resonant frequency change from 1.94 to $1.96 \mathrm{GHz}$ - a difference of $1.5 \%$ only.

S-Parameters $[\mathrm{dB}]$

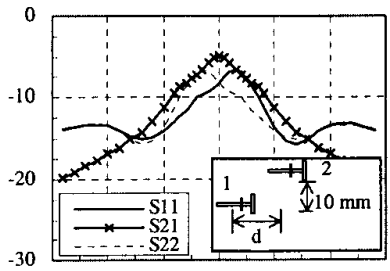

$-30$

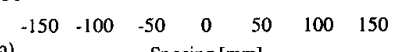

Spacing [mm]

Resonant frequency [GHz]

1,98

1,94

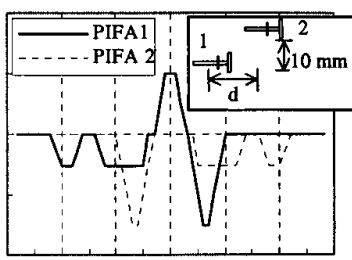

$\begin{array}{lllllll}-150 & -100 & -50 & 0 & 50 & 100 & 150\end{array}$

(b)

Spacing [mm]

Figure 2. Simulated S-parameters $S_{11}, S_{22}$ and $S_{21}$ (a) at the simulated resonant frequency (b) for two parallel PIFAs separated by $10 \mathrm{~mm}$ and fed at the same end, on infinite ground plane.

Similar simulations are made for the configuration where the two antennas are fed in the opposite ends $(0 ; 180)$; the results are shown in Figure 3. Except from the highest coupling that is obtained for $d=0$, the results are quite different from the $(0 ; 0)$, a rather unsymmetrical decrease is observed for an increase in the distance. This seems reasonable due to asymmetry in the setup. Similar to the $(0 ; 0)$ the coupling decreases almost linearly for $d=0$ to $150 \mathrm{~mm}$, whereas a rather different behaviour is observed for a negative $d$. A steep flange is observed where the feed lines are closest, i.e., for $d=-5$ to $-20 \mathrm{~mm}$, followed by an almost horizontal curve section where the coupling remains constant at around $-7 \mathrm{~dB}$ for $d=-20$ to $-40 \mathrm{~mm}$. Changing the horizontal distance such that the feed line is moved towards the shorting point causes a rapid decrease in the coupling, whereas moving the feed line away from the shorting point, i.e. towards the open end does not seem to affect the coupling. The coupling starts to decrease again when the two PIFAs are emancipated horizontally from each other. 


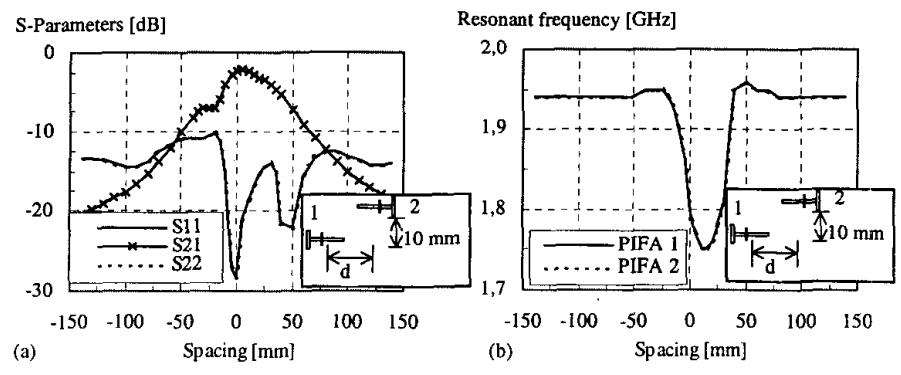

Figure 3. Simulated $S$-parameters $S_{11}, S_{22}$ and $S_{21}$ (a) at the simulated resonant frequency (b) for two parallel PIFAs separated by $10 \mathrm{~mm}$ and fed at the same end, on infinite ground plane.

The two identical reflection coefficients shown in Figure 3 (a) have two dips in the impedance match, the best at $d=0 \mathrm{~mm}$ and the other between $d=+40$ to $+50 \mathrm{~mm}$. The latter is when the vertical distance between the open ends are zero.

Comparing the simulated scattering parameters shown in Figure 2 (a) with 3 (a) it can be seen that the coupling is $2.5 \mathrm{~dB}$ higher for $(0 ; 180)$ than for $(0,0)$, this indicates lower coupling for the latter. However, making this comparison one should bear in mind that no correction have been made regarding the different impedance match. This explains parts of the difference. Nevertheless, it is the setup, which is shown in Figure 2 that yields the lowest coupling.

The resonant frequency, shown in Figure 3 (b), is altered quite dramatically. As for the $(0 ; 0)$ setup the largest deviation is obtained when the open end of one of the antennas is located above the feed line of the other; here the resonant frequency is decreased by $200 \mathrm{MHz}$. The resonant frequency seems almost symmetrically around the feed line, i.e., $d=13 \mathrm{~mm}$.

The results shown in Figure 2 and 3 are obtained using a finite number of samples, thus the results differs from the expected smooth curves.

B. Mutual Coupling versus Rotation of the Antennas for Fixed Distances: In the 26 setups that all together describe all cases of the mutual orientation, the simulated $S_{11}$ parameters show that the bandwidth, within which the reflection coefficient is better than $-6 \mathrm{~dB}$, is from 1.81 to $2.07 \mathrm{GHz}$, this is unchanged compared to the bandwidth for the single PIFA, which indicates a rather low coupling.

Using the geometrical centre of the PIFA as the centre of rotation makes it possible to define the two distances that is used in Figure 4. These are defined as the distance between the open ends of the two PIFAs and the distance between the shorting pins of the two PIFAs. The results differ from the expectations since the simulated coupling indicates that only $1.8 \mathrm{~dB}$ separates the lowest from the highest coupling. The highest coupling, $\mathrm{S}_{21}=-21.8 \mathrm{~dB}$ are found when the PIFAs are orientated such that the shorting pins are in the opposite ends, i.e., the maximum distance $(0 ; 180)$. The lowest coupling, $S_{21}=-23.6 \mathrm{~dB}$, are found when the shorting pins are located in the opposite ends, i.e., the minimum distance $(180 ; 0)$. Immediately, one would expect that the largest difference should be between a parallel and an orthogonal setup as for the Hertzian dipole.

C. Discussion of the results: To analyse the performance of the PIFA located above an infinite plane conductor, the image theory will be introduced to account for the reflections. The PIFA could be assumed to consist of two radiating components, namely the feed line (and the shorting pin) and the patch it self, the feed line being vertical, the patch horizontal. For an incident electric field with vertical polarisation, the polarisation of the reflected waves must also be vertical and with a polarity in the same direction as that of the actual source to satisfy the boundary conditions. For the radiating element in a horizontal orientation, it follows that the image is placed at a distance equal to the antenna height below the interface with a $180^{\circ}$ phase difference relative to the actual source. The radiation from the horizontal element and its 


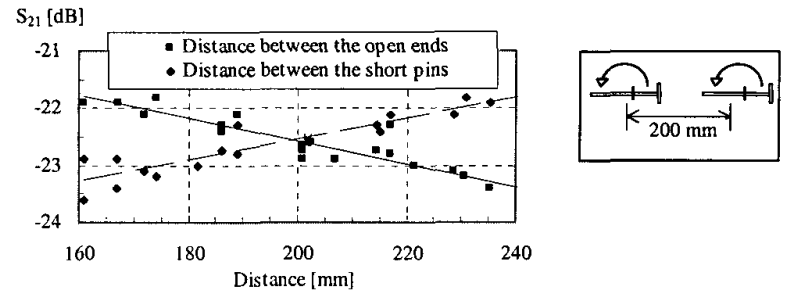

Figure 4. Simulated coupling as a function of the distance between the PIFA. The trend lines shown represent the coupling as a function of the distance between the open ends (solid), and the distance between the shorting pins (dashed).

image cancel in the plane at the ground plane; thus major parts of the radiated field originate from the feed line. This explains the rather low deviation in the field as a function of the mutual orientation, illustrated in Figure 4 . This is also in accordance with the fact that the highest coupling is found when the PIFAs are orientated such that the shorting pins are located in the opposite ends, i.e., maximum distance.

From a theoretical point of view a decrease in the field amplitude, hence coupling, is expected when the distance is increased, thus it seems reasonable that the distance between the open ends dictates the coupling. The results shown in Figure 4 support that.

Figure 4 also shows several points having the same distance but different coupling; this phenomenon is expected to be due to the polarisation of the PIFA.

The results for the coupling versus distance between the open ends and the shorting pins are shown in Figure 4. The results illustrate how to optimise the orientation and location to obtain minimum coupling. This result is in agreement with the results shown in section $A$.

V. ConClusions

The Planar Inverted F-Antenna (PIFA) has been characterised using IE3D. The prototype has a slightly higher bandwidth, mainly due to the cable used in the measurements and the simplifications used in the program model, whereas the resonant frequency is unchanged.

In accordance to the theory it is found that two identical PIFAs closely spaced affects each other. The resonant frequency is most affected at setups where the open end and the feed line are vertically on line. Maximum coupling is obtained when the antennas are overlapping each other, highest for the setup having the feed line in opposite ends.

For the setup of mutual rotation of the PIFAs the expected inverse proportionality between the coupling and the distance is not easily identified. However, the results states that it is most likely that it is the distance between the open ends of the PIFAs that dictates the coupling. Thus minimum coupling between two identical PIFAs is obtained when they are oriented such that the distance between the open ends is maximised; however the coupling mechanism is more complex than that.

\section{ACKNOWLEDGEMENT}

The author would like to thank K. B. Jakobsen (I), H. E. Gram (II), J. Troelsen (II), $\mathrm{O}$. Breinbjerg (I), and T. Olsgaard (II) for valuable discussions. (I) from Technical University of Denmark, (II) from Nokia Mobile Phones, Denmark.

\section{REFERENCES}

[1] Hirasawa, K., and Haneishi, M., "Analysis, design, and measurement of small and low profile antennas," Artech House, ISBN 0-89006-486-5, 1991.

[2] Appel-Hansen, J., "Centres of structures in electromagnetism - a critical analysis," IEEE Transaction on antennas and propagation, pp. 606-610, 1982.

[3] “IE3D User's Manual, Release 8", Zeland Software, Inc., Fremont, CA, 2001. 